\title{
Lung agenesis-heart defect-thumb anomalies syndrome
}

INSERM

\section{Source}

INSERM. (1999). Orphanet: an online rare disease and orphan drug data base. Lung agenesis-heart defect-thumb anomalies syndrome. ORPHA:1120

Lung agenesis - heart defect - thumb anomalies is a very rare syndrome characterized by unilateral complete or partial lung agenesis, congenital cardiac defects and ipsilateral thumb anomalies. 